\title{
Crescimento e fotossíntese de cana-de-açúcar em função de variáveis biométricas e meteorológicas
}

\author{
Ricardo A. Ferreira Junior ${ }^{1}$, José L. de Souza ${ }^{2}$, G ustavo B. Lyra ${ }^{3}$, \\ lêdo Teodoro ${ }^{1}, M \operatorname{arcos} A$. dos Santos ${ }^{1} \&$ Anthony C. S. Porfirio ${ }^{4}$
}

\begin{abstract}
RESU M 0
0 crescimento da cana-de-açúcar pode ser obtido por modelos biofísicos em que a fotossíntese bruta (FB) é obtida em função da radiação solar. 0 objetivo do trabalho é avaliar variedades de canas-deaçúcar em regime irrigado em relação à radiação fotossinteticamente ativa interceptada $\left(\right.$ RFA $\left._{\text {INT }}\right)$ e a estimativa da FB acumulada. Para isto, conduziu-se um estudo na U niversidade Federal de Alagoas, entre 2008 e 2009, com variedades de cana RB. Foram realizadas medidas biométricas, variáveis de produção e dos elementos meteorológicos. A irradiância fotossintética (RFA) interceptada foi obtida pela diferença entre RFA e RFA transmitida $\left(\mathrm{RFA}_{T}\right.$ ). A RFA foi determinada pela Lei de Beer. $\mathrm{N}$ a estimativa da FB diária usou-se uma integração numérica, com uma abordagem trapezoidal. As variáveis de produção tiveram correlações com a RFA INT $_{\text {T }}$ acumulada e com a FB acumulada durante o ciclo. A média da irradiação solar global diária do período chuvoso da região (maio - agosto) foi igual a $14,9 \mathrm{MJ} \mathrm{m}^{-2}$. A variedade RB92579 teve os maiores variáveis de produção, como também maiores RFA interceptada e FB acumuladas no ciclo, devido à sua maior capacidade de rebrotação e conversão de energia em fotoassimilados.
\end{abstract}

Palavras-chave: radiação fotossintética, modelos de crescimento, Saccharum spp.

\section{Growth and photosynthesis of sugarcane based on biometric and meteorological variables}

\begin{abstract}
Sugarcane growth can be obtained by biophysical models in which gross photosynthesis (GP) is obtained as a function of solar radiation. This work aims to evaluate sugarcane varieties under irrigation in relation to intercepted photosynthetic active radiation (PAR int) and the estimated accumulative GP. To achieve that, a study was conducted at the Federal U niversity of Alagoas during 2008 and 2009, with RB sugarcane varieties. Biometric measurements, production variables and meteorological elements were made. The intercepted photosynthetic irradiance (PAR) was obtained by the difference between PAR and transmitted PAR (PAR $)$, which was determined by Beer's Law. The daily GP was estimated numerically by the trapezoidal approach. The production variables had correlations with accumulated PARint and accumulated GP during the crop cycle. The average global solar radiation in the region for rainy season (M ay-August) was $14.9 \mathrm{MJ} \mathrm{m}^{-2}$. The variety RB92579 had the highest production variables as well as higher intercepted PAR and accumulated GP in the cycle due to its greater capacity for regrowth and energy conversion in photoassimilate.
\end{abstract}

Key words: photosynthetic radiation, growth models, Saccharum spp.

${ }^{1}$ CECA/U FAL, Campus Delza Gitaí, BR 101 - Norte, km 14, CEP 57100-000, Rio Largo, AL. E-mail: ricardo_ceca@hotmail.com; iedoteodoro@ig.com.br; marcosalex.ma@gmail.com

2 ICAT/U FAL, A. C. Simões, BR 104 - Norte, km 97, Tabuleiro dos Martins, CEP 57072-970, Maceió, AL. E-mail: jls@ccen.ufal.br

3 IF/D CA/U FRRJ, BR 465, Km 7, CEP 23890-970, Seropedica, RJ, E-mail: gblyra@gmail.com

${ }^{4}$ D SA/CPTEC/IN PE, Rod. Pres. D utra, km 39, CEP 12630-000, Cachoeira Paulista, SP. Fone: (21) 3186-9523. E-mail: anthony.carlos@cptec.inpe.br 


\section{INTRODUÇÃO}

O crescente incentivo pela utilização de energias de fontes renováveis eleva a demanda de combustíveis provenientes de biomassa vegetal (biocombustíveis), com destaque para o etanol da cana-de-açúcar (Saccharum spp.). Além disto, o setor sucroenergético gera também energia elétrica a partir dos resíduos da cana-de-açúcar (bagaço e palha). Outros produtos dessa cultura se sobressaem, tais como aguardente, vinhaça, plástico, o papel e o açúcar, que é o produto principal. A geração de energia renovável pelo cultivo de cana-de-açúcar pode ser convertida em créditos de carbono, pelo sequestro de $\mathrm{CO}_{2}$ da atmosfera. Desta forma, a área cultivada com cana se expande a cada ano; entretanto, em regiões canavieiras cujas áreas são restritas para expansão, é imprescindível o manejo adequado da cultura para alcançar produção potencial.

$\mathrm{O}$ entendimento da influência dos fatores ambientais no desenvolvimento e no crescimento de uma cultura é fundamental, uma vez que estão diretamente ligados a vários processos metabólicos (Monteith, 1977; Inman-Bamber, 1994). Assim, pesquisas direcionadas diversas vezes a fatores ambientais, interagem ou complementam as demais linhas de pesquisa ligadas à produção vegetal, em que o melhoramento vegetal é uma dessas linhas de pesquisa que analisam os efeitos dos fatores ambientais nas diferentes características morfológicas e fisiológicas de genótipos previamente selecionados, visando definir as variedades mais adequadas para uma localidade.

Estudos são dirigidos, visando à elaboração de modelos matemáticos para estimar o crescimento, o desenvolvimento e a produtividade das culturas (Jones \& Kiniry, 1986; Lisson et al., 2005; Oliveira et al., 2012). Em geral, esses modelos correlacionam os elementos ambientais com processos fisiológicos e com a produção das culturas, o que é possível em virtude da evolução do conhecimento dos processos fisiológicos vegetais e dos sistemas computacionais. A maioria dos modelos de crescimento considera a radiação solar como a variável principal de entrada (Keating et al., 1999; Liu \& Bull, 2001; Scarpari \& Beauclair, 2009), uma vez que esta variável é a fonte de energia para a fixação do $\mathrm{CO}_{2}$ atmosférico. Porém apenas uma porcentagem da radiação solar é utilizada na fotossíntese, que corresponde à parte da energia da faixa do espectro solar entre os comprimentos de onda de 400 a 700 nanômetros $(\mathrm{nm})$. Esta energia radiante é denominada radiação fotossinteticamente ativa (RFA), ou de luz. Nesses modelos a RFA interceptada $\left(\mathrm{RFA}_{\mathrm{INT}}\right)$ pelo dossel é função principalmente da radiação solar global incidente, do índice de área foliar (IAF) e do coeficiente de extinção da luz (K), em que o último expressa a influência das propriedades óticas das folhas e da geometria do dossel na atenuação da luz (Varlet-Grancher et al., 1989).

Em diversos modelos de simulação de crescimento de cultura, a influência da radiação solar é representada por um parâmetro conhecido como eficiência do uso da radiação(EUR), em que a espécie vegetal precisa ser cultivada sem limitações hídricas e/ou minerais. Nessas condições considera-se uma relação linear entre o acúmulo de matéria seca e a quantidade de radiação interceptada (Monteith, 1977). Os valores de EUR de plantas com mecanismo de fixação de carbono do tipo $\mathrm{C} 4$ variam de 3,0 a 4,0 g de fitomassa para cada MJ de RFA (Kiniry et al., 1989). Um método proposto por Monteith (1977) para estimar a taxa de crescimento da cultura (TCC) diária através da RFA $\mathrm{INT}_{\mathrm{IN}}$ da EUR, é de comprovada utilização, particularmente quando as condições ambientais são favoráveis ao crescimento potencial (Lizaso et al., 2005). Modelos como o CANEGRO e as primeiras versões do CERES-Maize usam este método para estimar a TCC diária. Este método, baseado no parâmetro EUR para representar o crescimento, tem limitações já que alguns dos principais processos fisiológicos que influenciam no crescimento das culturas (fotossíntese e a respiração) respondem diferentemente as variações das condições ambientais (Loomis \& Amthor, 1999; Lizaso et al., 2005).

Alguns modelos, como exemplo, o QCANE (Liu \& Bull, 2001; Singels et al., 2005), se preocupam com o detalhamento das relações dos fatores ambientais com os processos fisiológicos. Neste modelo o processo de crescimento da cultura inclui estimativas da fotossíntese e a respiração, que são funções do desenvolvimento da cultura e das variações dos elementos ambientes.

A computação da fotossíntese bruta de uma folha considerando-se a curva de resposta da assimilação de $\mathrm{CO}_{2}$ em função da RFA $_{\text {INT }}$ expressa uma forma hiperbólica (Goudriaan, 1986). Nos modelos existentes a fotossíntese é simulada após se contabilizar a variação de luz diária e da atenuação da luz dentro do dossel, ou seja, da luz interceptada, mais alguns parâmetros fotossintéticos da cultura, tais como a eficiência fotossintética e a fotossíntese máxima; este parâmetro expressa o caráter assintótico da taxa fotossintética. A equação usada com frequência para descrever esta relação é uma hiperbólica retangular, sendo ainda, base de muitos modelos de crescimento de cultura (Thornley, 1976).

Objetivou-se, com o presente trabalho, avaliar a produtividade de colmos ( $\mathrm{TCH}$ - tonelada de cana por hectare) e a de açúcar (TPH - tonelada de açúcar por hectare) de variedades RB de cana-de-açúcar irrigada por sistema de gotejamento subsuperficial em relação à RFA ${ }_{\mathrm{INT}}$ e a estimativa da fotossíntese bruta acumulada.

\section{MATERIAL E MÉTOdos}

A pesquisa foi conduzida com a primeira soca da cultura de cana-de-açúcar (RB92579, RB863129, RB931003, RB93509, RB72454, RB867515, RB951541, RB971755 e RB98710) na área experimental do Centro de Ciências Agrárias (CECA) da Universidade Federal de Alagoas (UFAL) em Rio Largo, Alagoas, Brasil ( $09^{\circ} 28^{\prime}$ 02" S; 35 49’ 43" W; 127 m) no período de fevereiro de 2008 a fevereiro de 2009. A área total de cultivo foi de $5.292 \mathrm{~m}^{2}$. O solo da área experimental foi classificado como Latossolo Amarelo Distrocoeso argissólico, de textura média/argilosa. $\mathrm{O}$ delineamento experimental foi em blocos casualizados, com quatro repetições. As parcelas foram constituídas de nove linhas de $15 \mathrm{~m}$. O espaçamento foi duplo (1,40 e $0,40 \mathrm{~m})$, sendo os toletes distribuídos de maneira que o arranjo fosse, em média, 18 gemas por metro linear. O cultivo foi irrigado por um sistema de gotejamento subsuperficial. Os dados agrometeorológicos (irradiância solar global, temperatura 
e umidade do ar, velocidade do vento) usados no estudo foram obtidos na estação agrometeorológica do CECA, localizada próxima à área experimental.

A evapotranspiração de referência (ETo) foi calculada pelo método de Penman-Monteith parametrizado no boletim FAO 56 (Allen et al., 1998). A evapotranspiração da cultura (ETc) foi calculada multiplicando-se ETo pelo coeficiente de cultura $(\mathrm{Kc})$. A variação do Kc nas fases de desenvolvimento foi definida através de observações do cultivo (cobertura foliar e altura de planta) e da estação do ano, seguindo-se a metodologia sugerida por Doorenbos \& Kassam (1979). O balanço hídrico (BH) em escala decendial (dez dias) foi feito pelo método de Thornthwaite \& Mather (1957), com capacidade de água disponível (CAD) no solo de $60,0 \mathrm{~mm}$, em uma profundidade efetiva do sistema radicular em torno de $0,60 \mathrm{~m}$, conforme dados físico-hídricos do solo da área da pesquisa. Foi considerada entrada de água no sistema, a precipitação pluvial mais a irrigação e, como saída, a ETc.

Os comprimentos e as larguras das folhas para determinação da área foliar (AF) da cultura foram obtidos em 12 plantas de cada variedade, nos seguintes dias, após o corte (DAC) da cana planta (20/02/08): 90, 128, 146, 176, 203, 245, 266, 299 e 336 dias. Nos mesmos dias avaliou-se o número de plantas por metro utilizando-se de $3 \mathrm{~m}$ lineares da mesma linha na qual estavam as plantas consideradas na determinação das dimensões foliares em cada parcela. O IAF foi obtido como a razão entre a $\mathrm{AF}$ e a área ocupada pela planta (AS). Para o cálculo do IAF diário no decorrer do cultivo tomaram-se os valores medidos e se ajustaram funções matemáticas em relação aos graus-dia acumulados ( $\Sigma \mathrm{GD})$ ao longo do cultivo. A equação ajustada foi a distribuição Log-Normal, com quatro parâmetros.

$$
y=a+b \exp \left[-0,5\left(\frac{\operatorname{Ln}\left(\frac{x}{c}\right)}{d}\right)^{2}\right]
$$

em que:

a - valor mínimo de y (IAF)

b - amplitude de y

c - valor de $\mathrm{x}(\Sigma \mathrm{GD})$ no qual yé máximo

d - grau de decaimento da variável y

Os cálculos dos GD foram realizados obedecendo a metodologia de Liu et al. (1998) para uma temperatura base de $20^{\circ} \mathrm{C}$.

A TCH foi determinada com auxílio de um dinamômetro com capacidade para $1.000 \mathrm{~kg}$, acoplado a uma carregadeira, nas duas linhas centrais de cada parcela ( $30 \mathrm{~m}$ linear). As análises da variável agroindustrial foram feitas no Laboratório da Usina Santa Clotilde, localizada em Rio Largo, AL. A TPH foi obtida através do produto entre a TCH e a POL de cana correspondente de cada parcela, dividida por cem. O TCH e o TPH das variedades foram submetidos à análise de variância.

A RFA foi estimada como $44 \%$ da irradiação solar global total diária $\left(\mathrm{H}_{\mathrm{g}}\right)$. Esta relação representa o valor médio determinado para a região de estudo. A $\mathrm{RFA}_{\mathrm{INT}}$ foi calculada por diferença, pela seguinte relação (Varlet-Grancher et al., 1989):

$$
\mathrm{RFA}_{\mathrm{INT}}=\mathrm{RFA}-\mathrm{RFA}_{\mathrm{T}}
$$

em que:

$\mathrm{RFA}_{\mathrm{T}}$ - RFA transmitida, estimada usando-se a equação similar a Lei de Beer:

$$
\mathrm{RFA}_{\mathrm{T}}=\mathrm{RFAexp}(-\mathrm{KIAF})
$$

em que:

$\mathrm{K}$ é adimensional. O coeficiente de extinção utilizado foi obtido de Inman-Bamber (1994), com valor igual a 0,58.

A estimativa da fotossíntese bruta diária $\left(\mathrm{FB}_{\mathrm{d}}, \mathrm{kg} \mathrm{m}^{-2} \mathrm{de}\right.$ $\mathrm{CO}_{2}$ ) foi feita através de uma solução numérica, baseada em uma abordagem trapezoidal, conforme metodologia de Liu (1996):

$$
\mathrm{FB}_{\mathrm{d}}=\frac{\mathrm{N}}{\mathrm{n}} \sum_{\mathrm{i}=0}^{\mathrm{n}-1}\left[\frac{\mathrm{FB}_{\text {INST }}\left[\operatorname{RFA}_{\text {INST }}\left(\mathrm{t}_{\mathrm{i}}\right)\right]+\mathrm{FB}_{\text {INST }}\left[\operatorname{RFA}_{\text {INST }}\left(\mathrm{t}_{\mathrm{i}}+1\right)\right]}{2}\right]
$$

em que:

taxa fotossintética bruta instantânea $\left(\mathrm{FB}_{\mathrm{INST}}, \mathrm{kg} \mathrm{m}^{-2} \mathrm{~s}^{-1} \mathrm{de}\right.$ $\mathrm{CO}_{2}$ )é obtida por:

$$
\mathrm{FB}_{\mathrm{INST}}=\left[\frac{\mathrm{F}_{\mathrm{MAX}}}{\mathrm{K}} \operatorname{Ln}\left(\frac{\alpha \operatorname{KRFA}_{\mathrm{INST}}\left(\mathrm{t}_{\mathrm{i}}\right)+(1-\tau) \mathrm{F}_{\mathrm{MAX}}}{\alpha \operatorname{KRFA}_{\text {INST }}\left(\mathrm{t}_{\mathrm{i}}\right) \mathrm{e}^{-\mathrm{KIAF}}+(1-\tau) \mathrm{F}_{\mathrm{MAX}}}\right)\right]
$$

em que:

$\alpha$ - eficiência fotossintética, $\mathrm{kg} \mathrm{J}^{-1} \mathrm{de} \mathrm{CO}_{2}$

$\mathrm{RFA}_{\mathrm{INST}}$ - irradiância fotossinteticamente ativa, $\mathrm{W} \mathrm{m}^{-2}$

$\mathrm{F}_{\mathrm{MAX}}$ - fotossíntese máxima, $\mathrm{kg} \mathrm{m}^{-2} \mathrm{~s}^{-1}$ de $\mathrm{CO}_{2}$, valor assintótico da taxa fotossintética em densidade de fluxo de luz saturante

$\mathrm{N}$ - fotoperíodo, s, calculado em função do dia do ano e a latitude

n - número de trapézios

$\tau \quad$ - coeficiente de transmissão $\left(\tau=\mathrm{RFA}_{\mathrm{T}} / \mathrm{RFA}\right)$

Os valores de $\alpha$ e $\mathrm{F}_{\mathrm{MAX}}$ para cana-de-açúcar foram, respectivamente, $10,88 \times 10^{-9} \mathrm{~kg} \mathrm{~J}^{-1} \mathrm{de} \mathrm{CO}_{2}$ e $1,57 \times 10^{-6} \mathrm{~kg} \mathrm{~m}^{-2} \mathrm{~s}^{-1} \mathrm{de} \mathrm{CO}_{2}$ (Liu, 1996; Hartt \& Burr, 1967).

Foram feitas correlações entre TCH com RFA ${ }_{\mathrm{INT}}$ e FB acumuladas no ciclo e entre TPH com RFA $\mathrm{INT}_{\mathrm{I}} \mathrm{e} \mathrm{FB}$, acumuladas no ciclo das variedades pesquisadas.

\section{RESULTADOS E DISCUSSÃO}

A precipitação pluvial foi, durante os 12 meses de cultivo, de $1.923 \mathrm{~mm}$ (com magnitude média de $10,2 \mathrm{~mm}$ por evento e frequência de aproximadamente um evento a cada dois dias), superando a ETc em 413 mm (Figura 1A); porém, devido à irregular distribuição temporal da chuva foi necessário aplicar uma irrigação de $699 \mathrm{~mm}$. AETc média diária foi de $4,3 \mathrm{~mm}$, valor próximo ao estimado por Lyra et al. (2007), para a mesma região de estudo, que foi de $4,6 \mathrm{~mm} \mathrm{~d}^{-1}$. Na Figura $1 \mathrm{~A}$ notou-se que em 
vários decêndios ocorreu elevada precipitação pluvial o que resultou na aplicação de apenas $61 \mathrm{~mm}$ de irrigação, entre os decêndios 5 (11-20/02/08) e 25 (01-10/09/08); no entanto, a partir do decêndio 26 (11-20/09/08), quando a estação seca da região se inicia (Souza et al., 2003), até o $1^{\circ}$ decêndio de 2009 (01-10/01/09), o consumo de água pela cultura foi maior que a precipitação pluvial, sendo necessária a aplicação de $637 \mathrm{~mm}$ via irrigação. Na fase final da cultura, necessitou-se de um período seco (irrigação suspensa em 07/01/09) para aumentar a concentração de sacarose nos colmos, porém ocorreu precipitação de $146 \mathrm{~mm}\left(40,6 \mathrm{~mm}\right.$ no $2^{\circ}$ decêndio de 2009 e $106,1 \mathrm{~mm}$ nos dois decêndios subsequentes 21/01-10/02/09); esta entrada de água no cultivo pressupõe-se ter influenciado negativamente a concentração de sacarose.

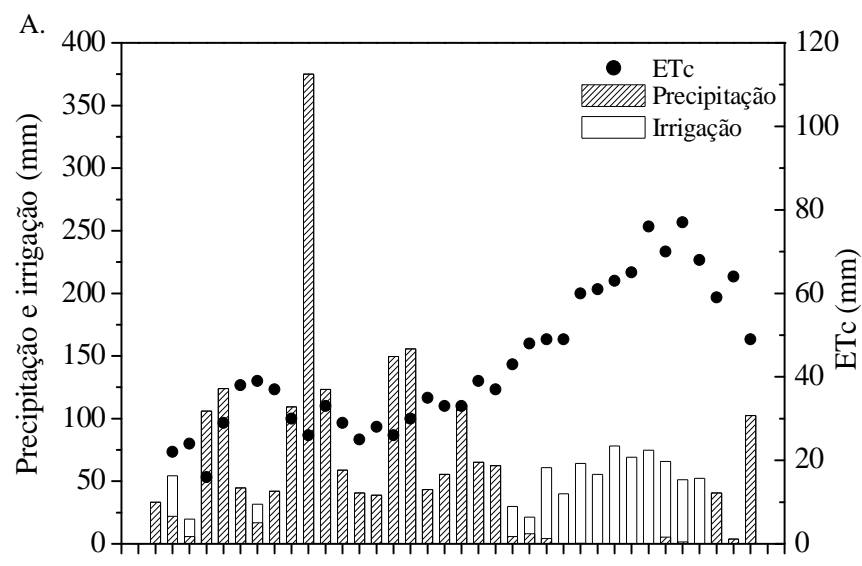

B.

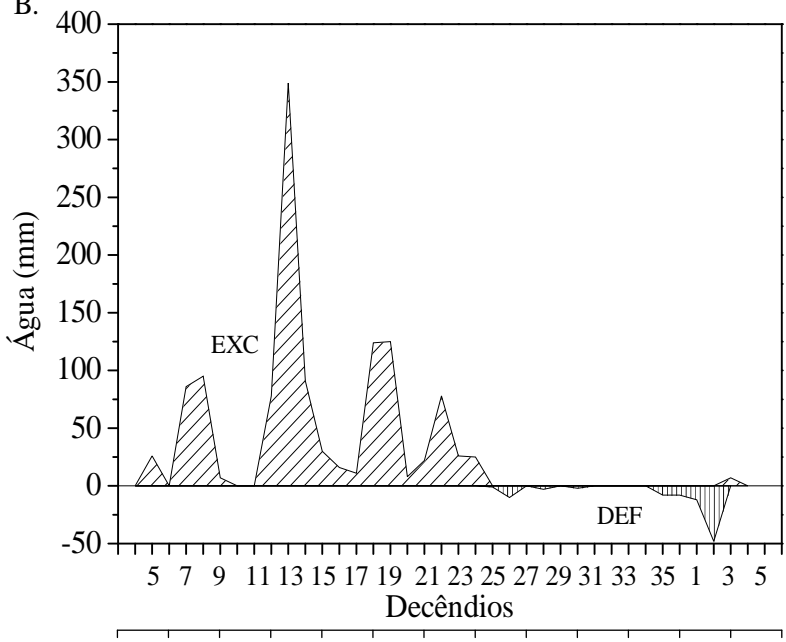

Fev Mar Abr Mai Jun Jul Ago Set Out Nov Dez Jan Fev Meses

Valores decendiais entre 01/02/2008 a 01/02/2009 em Rio Largo, AL

Figura 1. Precipitação pluvial, irrigação e evapotranspiração da cultura (ETC) (A); déficit (DEF) e excesso (EXC) hídrico no cultivo de cana irrigada ( $2^{\mathrm{a}}$ folha) (B)

O déficit hídrico total no cultivo foi de apenas $92 \mathrm{~mm}$ (Figura 1B), com $67 \mathrm{~mm}$ no primeiro decêndio de 2009, ou seja, no período em que a suspensão da irrigação ocorreu; logo, o cultivo não teve de maneira geral, restrição hídrica desfavorável aos processos de crescimento.

Durante o ciclo da cultura a média diária da temperatura do ar variou entre 20,1 e $28,2^{\circ} \mathrm{C}$ (Figura 2). Neste intervalo não

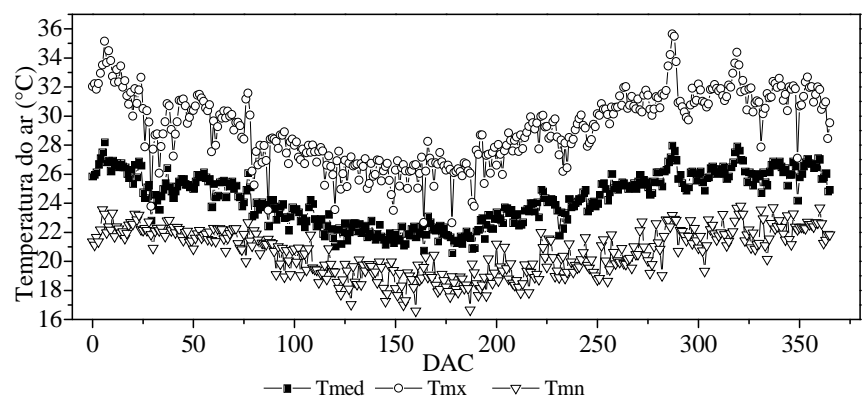

Figura 2. Temperatura do ar média (Tmed), mínima (Tmn) e máxima (Tmx) diária em função dos dias após o corte (DAC) no ciclo cultura da cana-de-açúcar, entre 01/ 02/2008 a 01/02/2009 em Rio Largo, AL

ocorreu limitação térmica para o desenvolvimento das plantas visto que, quando a média diária da temperatura do ar é igual ou superior $20^{\circ} \mathrm{C}$, tem-se aumento na taxa de crescimento da cultura sendo que o intervalo entre 25 e $33^{\circ} \mathrm{C}$ é considerado ótimo ao desenvolvimento vegetativo da cana-de-açúcar (Almeida et al., 2008). Os dias com média diária de temperatura do ar inferior a $20^{\circ} \mathrm{C}$, que ocorreram durante o ciclo da cultura (Figura 2), não ocasionaram perda de eficiência dos processos de crescimento, tal como a fotossíntese, uma vez que foram observados no período noturno. Pressupõe-se que as baixas temperaturas do ar ocorridas durante a noite proporcionaram ganhos, ao invés de causar perdas, já que ocorre diminuição do metabolismo (respiração) (Teh, 2006).

Os valores de IAF das variedades RB de cana-de-açúcar ao longo do ciclo, quando ajustados aos graus-dia acumulados ( $\Sigma \mathrm{GD}$ ) (Figura 3), foram bem estimados com $\mathrm{R}^{2}(0,81-0,96)$ (Tabela 1). As estimativas de IAF máximos variaram de 4,89 a 2,82, destacando-se a variedade RB93590, que apresentou o maior IAF máximo estimado (aos $930,7^{\circ} \mathrm{C} \mathrm{D}$ ), seguida da variedade RB98710 $\left(4,82\right.$ aos $976,3^{\circ} \mathrm{CD}$ ) (Figura 3). No entanto, essas variedades apresentaram maior taxa de diminuição dos valores máximos de IAF, até a colheita. Este padrão de decaimento do IAF na fase final da cultura é indicado pelo parâmetro "d" da equação Log-Normal, ou seja, quanto menor for o valor de "d" maior será o grau de decréscimo do IAF após o máximo (Tabela 1). Assim, é provável que, relativamente, as outras variedades, tenham, ao final do cultivo, interceptado quantidades similares de radiação fotossintética acumulada, tais como as variedades RB931003 e RB92579; apesar disto, a variedade RB92579, embora o IAF máximo $(3,84)$ tenha sido inferior aos das variedades RB98710 e RB93509, destacou-se pela maior interceptação de luz acumulada no ciclo, cujo desempenho ocorreu devido às elevadas taxas de crescimento do IAF no início do cultivo. Após o corte da cana-planta a variedade RB92579 apresentou a melhor capacidade de rebrota (com cerca de $23 \%$ a mais de brotos) comparada com as variedades RB92710 e RB867515 (segundo lugar em rebrota). Referidos maiores valores de IAF da variedade RB92579 no início do ciclo coincidem com altos fluxos de radiação solar o que, teoricamente, aumenta a diferença da radiação interceptada dessa variedade em relação às demais.

A análise de variância do IAF médio a nível de 5\% de probabilidade, apresentou diferença significativa entre as variedades e, de acordo com os resultados do teste de Tukey, 


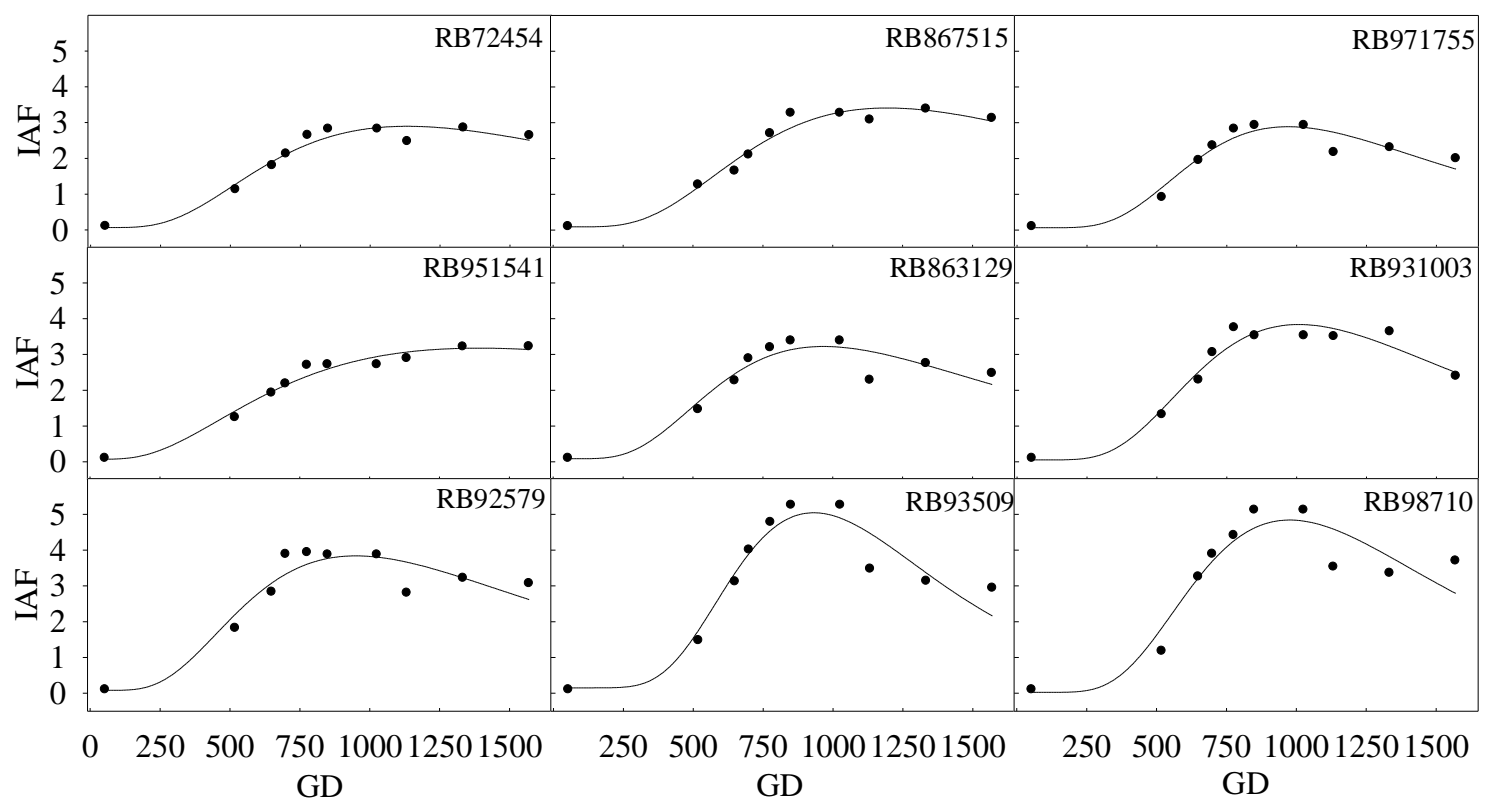

Figura 3. Ajuste entre o Índice de área foliar (IAF) de variedades RB de cana-de-açúcar e os graus-dia (G D) acumulados em cultivo ( $2^{\text {a }}$ fol ha) irrigado por gotejamento, entre 01/02/2008 a 01/02/2009 em Rio Largo, AL

Tabela 1. Parâmetros da equação Log-N ormal do ajuste entre o índice de área foliar (IAF) e os graus-dia acumulados ao longo do cultivo e IAF médio de variedades RB de cana-de-açúcar, irrigada por gotejamento

\begin{tabular}{lccrccc}
\hline Variedades & A & \multicolumn{1}{c}{ B } & \multicolumn{1}{c}{ C } & d & $\mathbf{R}^{\mathbf{2}}$ & IAF \\
RB971755 & 0,0662 & 2,8231 & 969,9 & 0,4616 & 0,8832 & $2,23 \mathrm{a}$ \\
RB72454 & 0,0680 & 2,8291 & 1132,2 & 0,5993 & 0,9272 & $2,35 \mathrm{ab}$ \\
RB951541 & 0,0742 & 3,1022 & 1391,3 & 0,7634 & 0,9591 & $2,59 \mathrm{abc}$ \\
RB863129 & 0,0863 & 3,1373 & 967,3 & 0,5328 & 0,8348 & $2,61 \mathrm{abc}$ \\
RB867515 & 0,0903 & 3,3220 & 1194,8 & 0,5625 & 0,9456 & $2,64 \mathrm{abc}$ \\
RB931003 & 0,0544 & 3,7827 & 1007,8 & 0,4736 & 0,9267 & $3,03 \mathrm{bcd}$ \\
RB92579 & 0,0825 & 3,7573 & 950,3 & 0,5663 & 0,8172 & $3,24 \mathrm{~cd}$ \\
RB98710 & 0,0266 & 4,8147 & 976,3 & 0,4508 & 0,8055 & $3,66 \mathrm{~d}$ \\
RB93509 & 0,1520 & 4,8926 & 930,7 & 0,3920 & 0,8693 & $3,68 \mathrm{~d}$ \\
\hline
\end{tabular}

Obs. Médias seguidas das mes mas letras não entre se diferem pelo teste de Tukey $(0,05)$

as variedades RB93509, RB931003, RB98710 e RB92579 não tiveram diferenças estatísticas significativas $(\mathrm{p}<0,05)$ entre si (Tabela 1) mas apresentaram tanto os maiores IAF médio como as maiores produtividades agrícolas.

A média diária da $\mathrm{H}_{\mathrm{g}}$ foi de $20,9 \mathrm{MJ} \mathrm{m}^{-2}$ do corte da cultura até 70 DAC (fevereiro - abril), período em que ocorreu a brotação (8 DAC) e se iniciou o crescimento da área foliar, em razão de que a média diária da $\mathrm{RFA}_{\mathrm{INT}}$ da variedade RB92579 (variedade de maior interceptação no ciclo) foi baixa nesta fase inicial (1,9 $\mathrm{MJ} \mathrm{m}^{-2}$ ) no dia 30/04/09 (70 DAC), quando o IAF dessa variedade apresentou valor igual a 1,45 e acumulou, até esse dia, $121,3 \mathrm{MJ} \mathrm{m}^{-2}$ de RFA $_{\mathrm{INT}}$. Nesta ocasião, a variedade RB92579 apresentou a maior fração de $\mathrm{RFA}_{\mathrm{INT}}\left(\mathrm{fRFA}_{\mathrm{INT}}\right)$ entre as variedades, ou seja, de 0,57, enquanto a variedade RB971755 mostrou a menor fRFA $\mathrm{INT}(0,31)$.

Apesar da baixa interceptação de luz de fevereiro a abril (70 DAC) em relação aos valores posteriores do ciclo, a média da $\mathrm{H}_{\mathrm{g}}$ diária foi elevada quando comparada com a do período chuvoso da região, maio - agosto (71 - 193 DAC), que apresentou valor médio de $14,9 \mathrm{MJ} \mathrm{m}^{-2}$ (Figura 4). Os menores valores de $\mathrm{H}_{\mathrm{g}}$ nos meses chuvosos foram devidos

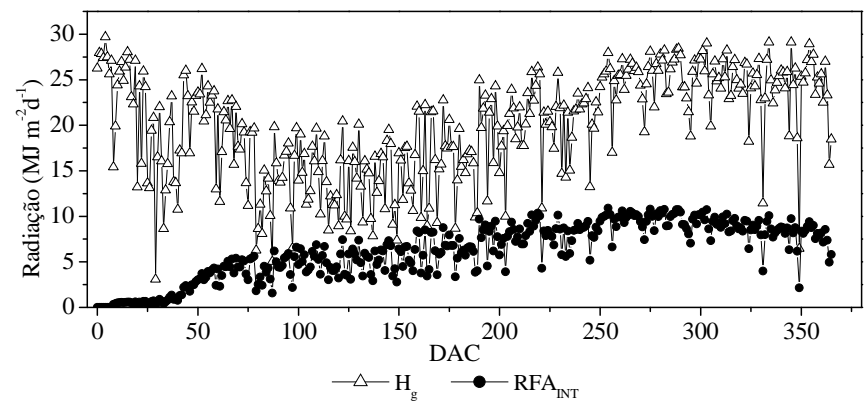

Figura 4. Irradiação solar global diária $\left(\mathrm{H}_{\mathrm{q}}\right)$ e irradiação fotossinteticamente ativa interceptada $\left(\operatorname{RFA}_{\text {INT }}\right)$ da variedade RB92579 em função dos dias após o corte (DAC) durante o ciclo cultura da cana-de-açúcar, em Rio Largo, AL

principalmente à elevada nebulosidade, associada ao aumento do ângulo zenital dessa época do ano. Souza et al. (2005) relatam que a irradiação solar global em dias de céu limpo varia em $25 \%$ entre o período chuvoso e o seco da região em estudo; com isto, no período de alta demanda energética da cultura (fase de crescimento vegetativo intenso), a interceptação absoluta de luz pelas plantas não é maximizada por conta da redução da radiação solar incidente, haja vista que o aparato interceptador (área foliar) apresentou crescimento contínuo ao longo deste período. A variedade RB93509 apresentou o maior $\operatorname{IAF}(4,79)$ e as variedades RB951541 e RB72454, os menores $(2,51)$.

Durante o período chuvoso a amplitude da fração da RFA interceptada ou $\mathrm{fRFA}_{\mathrm{INT}}$ das variedades foi 0,32 (RB971755) a 0,95 (RB98710) a última, conseguida no final do período (193 DAC), é considerada o completo fechamento da cobertura vegetal. Em suas pesquisas, Robertson et al. (1996), em Queensland, Austrália $\left(18,7^{\circ} \mathrm{S}\right.$ e $\left.146,2^{\circ} \mathrm{E}\right)$, reportaram que o completo fechamento do dossel (>0,95 de radiação interceptada) foi alcançado em 150 DAC, sem limitação de água, sinalizando que a variedade Australiana teve relativo maior 
potencial de interceptação da radiação solar. Inman-Bamber \& Smith (2005) relatam que a fRFA interceptada foi 0,92 quando a cultura tinha um IAF igual a 3,0.

A média diária de $\mathrm{RFA}_{\mathrm{INT}}$ do período chuvoso das variedades foi entre 4,28 (RB72454) a 5,33 $\mathrm{MJ} \mathrm{m}^{-2}$ (RB92579 e RB93509). A variedade RB92579 continuou sendo a que mais interceptou luz, com acúmulo de $776,5 \mathrm{MJ} \mathrm{m}^{-2}$ de $_{\text {RFA }}$ e a variedade RB971755 a que apresentou menor acúmulo do início do cultivo até o período (193 DAC) somando 587,7 $\mathrm{MJ} \mathrm{m}^{-2}$. O período do ciclo da cultura com maior média diária de $\mathrm{RFA}_{\mathrm{INT}}$ (7,49 a 9,02 $\mathrm{MJ} \mathrm{m}^{-2}$ ) ocorreu entre 194 e 365 DAC (colheita); esta alta média foi resultante da elevada média diária de $\mathrm{H}_{\mathrm{g}}$ $\left(23,3 \mathrm{MJ} \mathrm{m}^{-2}\right)$ e da maior fração de $\mathrm{RFA}_{\text {INT }}$, que variou de $0,73^{\mathrm{g}}$ (RB971755) a 0,88 (RB98710).

As variedades RB92579 e RB98710 foram as que tiveram maior TCH (produtividade agrícola) com médias de 154,63 ( \pm $10,13)$ e $139,81( \pm 10,12)$ t ha $^{-1}$, respectivamente (Tabela 2) porém entre as variedades avaliadas não existiu diferença estatística significativa para o TCH, pela ANOVA, a nível de 5\% de probabilidade. Na avaliação da TPH (produtividade de açúcar) houve amplitude elevada entre as variedades $\left(9,60 \mathrm{t} \mathrm{ha}^{-1}\right)$, em que a variedade RB863129 teve o menor valor $\left(16,61 \mathrm{tha}^{-1}\right)$ e a RB92579 se destacou com 26,21 t ha-1 de açúcar (Tabela 2). Todavia, pela análise de variância a nível de 0,05 de probabilidade não se observaram diferenças estatísticas significativas entre as variedades em estudo.

Tabela 2. Produtividade de colmos $(\mathrm{TCH})$, produtividade de açúcar (TPH), irradiação fotossinteticamente ativa interceptada $\left(\mathrm{RFA}_{\mathrm{INT}}\right)$, e fotossíntese bruta (FB) para as variedades RB de cana-de-açúcar em cultivo irrigado por gotejamento

\begin{tabular}{|c|c|c|c|c|}
\hline \multirow{2}{*}{ Variedades } & TCH & TPH & \multirow{2}{*}{$\begin{array}{c}\text { RFA }_{\mathrm{INT}} \\
\left(\mathrm{MJ} \mathrm{m}^{-2}\right)\end{array}$} & \multirow{2}{*}{$\begin{array}{c}F B \\
\left(\mathrm{~kg} \mathrm{~m}^{-2}\right)\end{array}$} \\
\hline & \multicolumn{2}{|l|}{$\left(t\right.$ ha $\left.^{-1}\right)$} & & \\
\hline RB863129 & $109,88 \pm 4,37$ & 16,61 & $2.075,07$ & 16,30 \\
\hline RB951541 & $114,81 \pm 6,65$ & 18,62 & $2.098,24$ & 16,46 \\
\hline RB72454 & $116,67 \pm 9,07$ & 18,42 & $1.995,55$ & 15,22 \\
\hline RB971755 & $120,99 \pm 10,43$ & 18,72 & $1.875,97$ & 14,98 \\
\hline RB867515 & $124,69 \pm 8,60$ & 20,29 & $2.080,21$ & 16,32 \\
\hline RB93509 & $133,33 \pm 10,25$ & 21,54 & $2.223,42$ & 17,13 \\
\hline RB931003 & $134,57 \pm 6,76$ & 21,66 & $2.128,47$ & 16,53 \\
\hline RB98710 & $139,81 \pm 10,12$ & 23,59 & $2.260,75$ & 17,26 \\
\hline RB9257/9 & $154,63 \pm 10,13$ & 26,21 & $2.262,21$ & 17,47 \\
\hline
\end{tabular}

\pm erro padrão de quatro repetições

A variedade RB92579 também foi a que obteve a maior estimativa da $\mathrm{FB}\left(17,46 \mathrm{~kg} \mathrm{~m}^{-2} \mathrm{de} \mathrm{CO}_{2}\right)$ seguida, novamente, da variedade RB98710, que teve fixação bruta de $\mathrm{CO}_{2}$ de 17,26 $\mathrm{kg} \mathrm{m}^{-2}$. A RFA ${ }_{\mathrm{INT}}$ acumulada nos ciclos dessas variedades foi similar, com diferença menor que $2,0 \mathrm{MJ} \mathrm{m}^{-2}$ (Tabela 2). Do início do cultivo até o 115 dias após o corte (período em que as plantas da variedade RB92579 tinham valores de IAF superior aos apresentados pela RB98710) o dossel da RB92579 interceptou $81,76 \mathrm{MJ} \mathrm{m}^{-2}$ a mais que o da RB98710. Em relação à FB acumulada sua diferença foi de 426,02 $\mathrm{g} \mathrm{m}^{-2}$ entre as mesmas variedades, sendo maior na RB92579. A média da $\mathrm{H}_{\mathrm{g}}$ neste período foi elevada com valor de 18,42 $\mathrm{MJ} \mathrm{m}^{-2}$. A partir dos 116 dias após o corte, a variedade RB98710 interceptou mais RFA que a RB92579, o que resultou em acúmulo de RFA $_{\mathrm{INT}}$ similar para as duas variedades, ao final do ciclo; todavia, o total de FB e a TCH da variedade RB92579 foram superiores aos obtidos pela RB98710.

Como a densidade de fluxo de luz interceptado pelo dossel é maior quando as plantas têm IAF elevado, a fotossíntese máxima (saturação da fotossíntese) minimizou a diferença da estimativa de FB entre as variedades RB98710 e RB92579, após 116 DAC até o fim do ciclo, período no qual a RB98710 teve $\mathrm{IAF} \mathrm{RFA}_{\mathrm{INT}}$ superiores a RB92579 e, consequentemente, maior FB; entretanto, não foi suficiente para superar a diferença que a RB92579 obteve no início do ciclo; assim, mesmo as variedades interceptando valores totais de luz próximos, a variedade RB92579 teve acentuada vantagem de fixação bruta de $\mathrm{CO}_{2}$ acumulada ao longo do ciclo, relacionada principalmente com a capacidade de rebrotamento inicial.

A estimativa da produtividade de colmos (considerando-se todas as variedades) em função da RFA $_{\mathrm{INT}}$ acumulada, através de regressão linear forçada a passar pela origem, apresentou coeficiente de determinação de 0,51 e coeficiente angular de 0,0061 ( $\pm 0,0002)$ (Figura 5A). Quando foi excluída da análise a variedade $\mathrm{RB} 971755 \mathrm{o} \mathrm{R}^{2}$ foi de 0,71 e o coeficiente angular de $0,0060( \pm 0,0002)$ ou seja, em média para cada $\mathrm{MJ}$ de radiação fotossintética interceptada no ciclo da cultura proporciona um ganho de aproximadamente $6 \mathrm{~g}$ de colmos frescos por $\mathrm{m}^{2}$. Considerando proporções entre a biomassa seca total, colmos frescos e secos relatadas por Robertson et al. (1999), este valor equivale a uma EUR de aproximadamente $0,8 \mathrm{~g} \mathrm{MJ}^{-1}$ de biomassa seca total usando-se a radiação solar global interceptada. Singels et al. (2005) mencionam que cultivos em Pongola têm valores de EUR variando ente 0,8 a 1,2 $\mathrm{g} \mathrm{MJ}^{-1}$. A variedade RB971755 apresentou padrão diferenciado uma vez que, mesmo com a

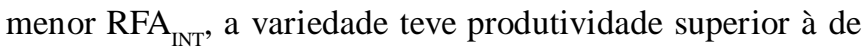
três variedades que tiveram maior interceptação de luz (RB863126, RB951541 e RB72454), evidenciando que a variedade RB971755 tem melhor eficiência de conversão da radiação solar em biomassa.

A regressão linear (forçada a passa pela origem) entre a produtividade de colmos e a fotossíntese bruta acumulada ao longo do ciclo, teve um $\mathrm{R}^{2}$ de 0,49 e coeficiente angular de $0,7764$ ( $\pm 0,0219)$ (Figura 5B); no entanto, algumas variedades (RB863129, RB72454 e RB971755) apresentaram padrões diferenciados das demais; assim, quando a análise foi feita sem as variedades RB863129, RB72454 e RB971755, o R 2 apresentou acréscimo, com valor igual a 0,72 e coeficiente angular similar $(0,7732)$.

A estimativa da produtividade de açúcar $\left(\mathrm{kg} \mathrm{m}^{-2}\right)$ das variedades $R B$ em função da $R_{\text {FANT }}$ acumulada $\left(\mathrm{MJ} \mathrm{m}^{-2}\right)$, através de uma regressão linear forçada a passar pela origem, apresentou $R^{2}$ de 0,57 e coeficiente angular de $0,0010( \pm 0,00003)$ (Figura 6A) o que, em média, cada MJ interceptado a mais por um $\mathrm{m}^{2}$ de plantas de cana-de-açúcar irrigada aumenta um grama de açúcar; a produtividade de açúcar também pode ser estimada pela FB acumulada, por um fator de $0,1256( \pm 0,0048)$ e $\mathrm{R}^{2} \mathrm{de}$ 0,55 (Figura 6B). 

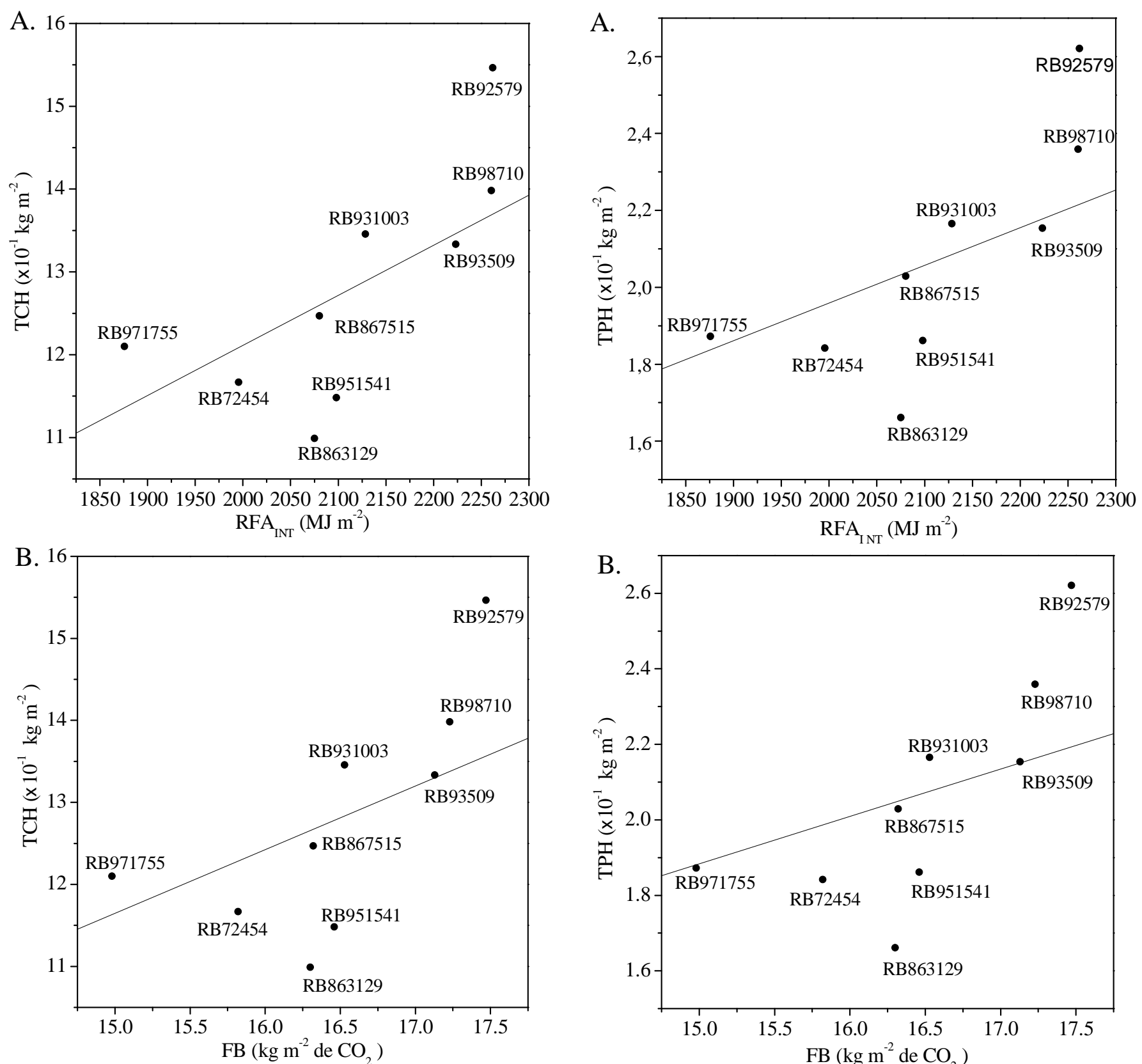

Figura 5. Correlação entre a produtividade de colmos (TCH ): e a radiação fotossinteticamente ativa interceptada $\left(\mathrm{RFA}_{\mathrm{INT}}\right)$ acumulada (A); a fotossíntese bruta (FB) acumulada no cultivo de variedades RB de cana-de-açúcar $\left(2^{\circ}\right.$ folha) irrigado por gotejamento entre $01 / 02 / 2008$ a $01 / 02 / 2009$ (B)

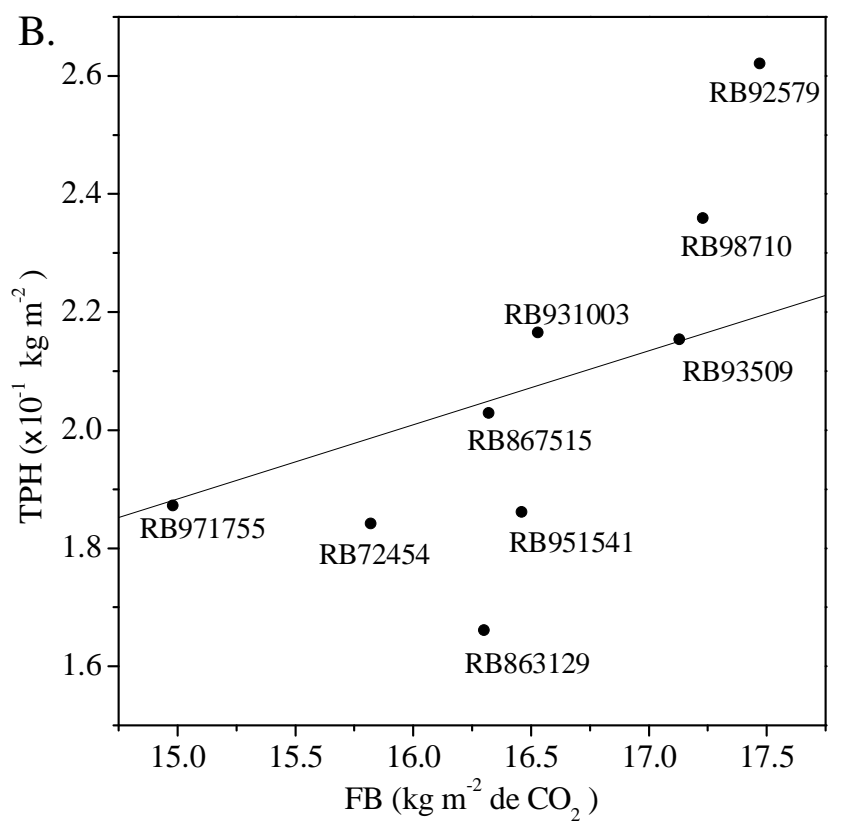

Figura 6. Correlação entre produtividade de açúcar (TPH): e a radiação fotossintética interceptada $\left(\mathrm{RFA}_{\mathrm{INT}}\right)$ acumulada (A); a fotossíntese bruta (FB) acumulada no cultivo de variedades RB de cana-de-açúcar $\left(2^{\circ}\right.$ folha) irrigado por gotejamento entre $01 / 02 / 2008$ a 01/02/2009 (B)

\section{CONCLUSÕES}

1. As variedades $\mathrm{RB}$ de cana-de-açúcar irrigada por gotejamento subsuperficial nos Tabuleiros Costeiros de Alagoas apresentam tendência crescente das correlações entre as produtividades (de colmos e de açúcar) e a radiação fotossinteticamente ativa acumulada e com a fotossíntese bruta acumulada durante o ciclo.

2. A variedade RB92579 é a que se destaca com maiores rendimentos de colmos e de açúcar devido à sua maior capacidade de rebrotação e eficiência em conversão de energia (radiação fotossinteticamente ativa) em fotoassimilados.

\section{LITERATURA CITADA}

Allen, R. G.; Pereira, L. S.; Raes, D.; Smith, M. Crop evapotranspiration: Guidelines for computing crop water requirements. Rome: FAO 1998. 300p. Irrigation and Drainage, Paper, 56.

Almeida, A. C. S.; Souza, J. L.; Teodoro, I.; Barbosa, G. V. S.; Moura Filho, G.; Ferreira Júnior, R. A. Desenvolvimento vegetativo e produção de variedades de cana-de-açúcar em relação à disponibilidade hídrica e unidades térmicas. Ciência e Agrotecnologia, v.32, p.1441-1448, 2008. 
Doorenbos, J.; Kassam, A. H. Yield response to water. Rome: Food and Agriculture Organization of the United Nations, 1979. 193p. Irrigation and Drainage Paper, 33

Goudriaan, J. A simple and fast numerical method for the computation of daily total photosynthesis. Agricultural and Forest Meteorology, v.38, p.249-254, 1986.

Hartt, C. E.; Burr, G. O. Factors affecting photosynthesis in sugarcane. In: International Society Sugarcane Technologists, 12, San Juan, 1965. Proceedings... Amsterdam: ISSCT, 1967. p.590-609.

Inman-Bamber, N. G. Temperature and seasonal effects on canopy development and light interception of sugarcane. Field Crops Research, v.36, p.41-51, 1994.

Inman-Bamber, N. G.; Smith, D. M. Water relations in sugarcane and response to water deûcits. Field Crops Research, v.92, p.185-202, 2005.

Jones, C. A.; Kiniry, J. R. CERES-Maize: A simulation model of maize growth and development. Texas: Texas A \& M University Press, College Station, 1986. 194p.

Keating, B. A.; Robertson, M. J.; Muchow, R. C.; Huth, N. I. Modelling sugarcane production systems I: Development and performance of the sugarcane module. Field Crops Research, v.48, p.27-36, 1999.

Kiriny, J. R.; Jones, C. A.; O’Toole, J. C.; Blanchet, R.; Cabelguenne, M.; Spanel, D. A. Radiation-use efficiency in biomass accumulation prior to grain-filling for five graincrop species. Field Crops Research, v.20, p.51-64, 1989.

Lisson, S. N.; Inman-Bamber, N. G.; Robertson, M. J.; Keating, B. A. The historical and future contribution of crop physiology and modeling research to sugarcane production systems. Field Crops Research, v.92, p.321-335, 2005.

Liu, D. L. Incorporating diurnal light variation and canopy light attenuation into analytical equations for calculating daily gross. Ecology Modelling, v.93, p.175-189, 1996.

Liu, D. L.; Bull, T. A. Simulation of biomass and sugar accumulation in sugarcane using a process-based model. Ecological Modelling, v.144, p.181-211, 2001.

Liu, D. L.; Kingston, G.; Bull, T. A. A new technique for determining the thermal parameters of phenological development in sugarcane, including sub-optimum and supra-optimum temperature regimes. Agricultural and Forest Meteorology, v.90, p.119-139, 1998.

Lizaso, J. I.; Batchelor, W. D.; Boote, K. J.; Westgate, M. E. Development of a leaf-level canopy assimilation model for CERES - Maize. Agronomy Journal, v.97, p.722-733, 2005.

Loomis, R. S.; Amthor, J. S. Yield potential, plant assimilatory capacity, and metabolic efficiencies. Crop Science, v.39, p.1584-1596, 1999.
Lyra, G. B.; Sediyama, G. C.; Lyra, G. B.; Pereira, A. R.; Souza, E. F. de. Evapotranspiração da cultura de cana-de-açúcar na região de tabuleiros costeiros do estado de Alagoas: Coeficiente de cultura "único" padrão boletim FAO - 56. Sociedade dos Técnicos Açucareiros e Alcooleiros do Brasil -STAB, v.25, p.40-43, 2007.

Monteith, J. L. Climate and the efficiency of crop production in Britain. Philosophical Transactions Royal, v.281, p.277-294, 1977.

Oliveira, R. A.; Santos, R. S.; Ribeiro, A.; Zolnier, S.; Barbosa, M. H. P. Estimativa da produtividade da cana-de-açúcar para as principais regiões produtoras de Minas Gerais usandose o método ZAE. Revista Brasileira de Engenharia Agrícola eAmbiental, v.16, p.549-557, 2012.

Robertson, M. J.; Inmam-Bamber, N. G.; Muchow, R. C.; Wood, A. W. Physiology and productivity of sugar cane with early and mid-season water deficit. Field Crops Research, v.64, p.211-227, 1999.

Robertson, M. J.; Wood, A. W.; Muchow, R. C. Growth of sugarcane under high input conditions in tropical Australia: I. Radiation use, biomass accumulation and partitioning. Field Crops Research, v.48, p.11-25, 1996.

Scarpari, M. S.; Beauclair, E. G. F. Physiological model to estimate the maturity of sugarcane. Scientia Agricola. v.66, p.622-628, 2009.

Singels, A.; Donaldson, R. A.; Smit, M. A. Improving biomass production and partitioning in sugarcane: Theory and practice. Field Crops Research, v.92, p.291-303, 2005.

Souza, J. L.; Moura Filho, G.; Lyra, R. F. F.; Teodoro, I.; Santos, E. A.; Silva, J. L. Silva, P. R. T.; Cardim, A. H.; Amorim, C. A. Análise da precipitação pluvial e temperatura do ar na região do Tabuleiro Costeiro de Maceió, AL, período 1972-2001. Revista Brasileira de Agrometeorologia, v.11, p.131-141, 2003.

Souza, J. L.; Nicácio, R. M.; Moura, M. A. L. Global solar radiation measurements in Maceió, Brazil. Renew Energy, v.30, p.1205-1220, 2005.

Teh, C. B. S. Introduction to mathematical modeling of crop growth: How the equations are derived and assembled into a computer program. Boca Raton: Brown Walker Press, 2006. 256p.

Thornley, J. H. M. Mathematical models in plant physiology. London: Academic Press, 1976. 318p.

Thornthwaite, C. W. Mather, J. R. Instructions and tables for computing potencial evapotranspiration and the water balance. Ceterton: Rexel Institute of Technology-Laboratory, 1957. 311p.

Varlet-Grancher, C.; Gosse, G.; Chartier, M.; Sinoquet, H.; Bonhomme, R.; Allirand, J. M. Mise au point: Rayonnement solaire absorbé ou intercepté par un couvert végétal. Agronomie, v.9, p.419-439, 1989. 